\title{
THE GEOMETRIC MEAN CONCURRENT CONVERGENCE METHOD
}

\author{
Takao Ohya* \\ School of Science and Engineering \\ Kokushikan University \\ Setagaya, Tokyo, Japan \\ E-mail: takaohya@kokushikan.ac.jp \\ Eizo Kinoshita \\ Faculty of Urban Science \\ Meijyo University \\ Kani, Gifu, Japan \\ E-mail: kinoshit@urban.meijo-u.ac.jp
}

\begin{abstract}
This study develops an error model of dominant AHP, and the geometric mean concurrent convergence method (GMCCM). The dominant AHP was originally developed by Kinoshita and Nakanishi. The dominant AHP focuses on a specific alternative and refers to the relative importance of the criteria from the specific alternative. The specific alternative is called the regulating alternative. Furthermore, Kinoshita and Nakanishi developed the concurrent convergence method (CCM) for the case that several regulating alternatives exist and relative importance from each regulating alternative is inconsistent. Kinoshita, Sekitani and Shi proved the convergence of CCM. In this study, a multiplicative error model for the case where several regulating alternatives exist and are inconsistent is made and then, from leastsquare regression of that model, GMCCM is derived.
\end{abstract}

Keywords: dominant AHP, multiplicative error model, geometric mean concurrent convergence method

\section{Dominant AHP}

AHP is a flexible decision making system that can deal with the subjective judgments of a decision maker. Its numerously successful applications have been reported in this field [1]. In AHP, the decision maker identifies an ambiguous evaluation problem into a hierarchy structure within the evaluation goal, criteria and alternatives, each of which corresponds to a node of the hierarchy. A hierarchy with a top, middle and bottom structure usually consists of three levels; the goal, the criteria, and the alternatives, respectively. Directed arcs of the hierarchy form a parent-child relationship among the nodes and the existence of a pair of parent-child nodes means that the decision maker judges the relative importance of the child-nodes from the parent-node. That is, for example, directed arcs from the top level to the middle level indicate the decision maker's judgment on the relative importance of all criteria from the goal. Saaty [1] proposes that in this three-level hierarchy the decision maker first judges the relative importance of the criteria from the goal and, secondarily, judges that of the alternative from the criteria. Judgments of the relative importance are expressed numerically, which are called evaluation values. Let $\boldsymbol{A}$ and $\boldsymbol{C}$ be a set of alternatives and that of criteria, respectively, and denote their cardinalities by $A$ and $C$, respectively. Then we have a total of $|A| \times(C+1)$ evaluation values in the three-level hierarchy. By plotting a set of evaluation values on the arcs of hierarchy, the hierarchy becomes a network tree with the directed arcs. In the original AHP, the evaluation value of a child-node from a parent-node is quantified under the assumption that the decision maker compares all pairs between distinct two children of a parent.

\footnotetext{
${ }^{*}$ Corresponding author
} 
Kinoshita and Nakanishi [2] focus on the following empirical result: When the decision maker evaluates relative importance of the criteria from the goal, he/she focuses on a specific alternative and refers to the relative importance of the criteria from the specific alternative. The specific alternative is called the regulating alternative. Kinoshita and Nakanishi [2] assume that if there exists exactly one regulating alternative, then the relative importance of the criteria from the regulating alternative determines that from other alternatives. If there exists only one regulating alternative in the alternative set, then the regulating alternative is called the dominant one and they implement the assumption into the dominant AHP. The mathematical description of the dominant AHP is as follows:

\section{Algorithm: Dominant AHP}

Step 0: The decision maker selects a regulating alternative from the alternative set $\boldsymbol{A}$. Let alternative $r$ be the regulating alternative.

Step 1: From the viewpoint of every criterion $c \in C$, the decision maker evaluates the relative importance of all alternatives and quantifies the evaluation values of all alternatives. Let $v_{a c}$ be the evaluation value of the alternative $a$ from the criterion $c$.

Step 2: From the viewpoint of the regulating alternative $r$, the decision maker evaluates the relative importance of all criteria and quantifies the evaluation values of all criteria. Let $w_{c}^{r}$ be the evaluation value of the criterion $c$ from the regulating alternative $r$.

Step 3: Let $u_{a c}^{r}$ denote the evaluation value of the alternative $a$ from the criterion $c$ normalized by the evaluation value of the alternative $r$ from the criterion $c$.

$$
u_{a c}^{r}=v_{a c} / v_{r c}
$$

Step 4: Let $t_{a}$ denote the overall evaluation value of alternative $a$, which is the summation of products of $u_{a c}^{r}$ by $w_{c}^{r}$.

$$
t_{a}=\sum_{c} w_{c}^{r} u_{a c}^{r}
$$

\section{CCM}

Suppose that the alternative $r$ is the dominant one. Let $\hat{\mathbf{w}}^{r\left(r^{\prime}\right)}$ be a $|\boldsymbol{C}|$-dimensional vector whose $c$ th element $\widehat{w}_{c}^{r\left(r^{\prime}\right)}$ is an unknown evaluation value of the criterion $c$ from the alternative $r \neq r^{\prime}$. Then, Kinoshita and Nakanishi [2] propose the following evaluation rule under their assumption.

$$
\widehat{w}_{c}^{r\left(r^{\prime}\right)}=\frac{w_{c}^{r^{\prime}} v_{r c} / v_{r^{\prime} c}}{\sum_{b} w_{b}^{r^{\prime}} v_{r b} / v_{r^{\prime} b}}
$$

In CCM [2] the decision maker selects some alternatives that play the role of yardsticks in evaluation process. Let $\boldsymbol{R}$ be a set of regulating alternatives. CCM requires the decision maker to evaluate criteria from the viewpoint of each regulating alternative $r \in \boldsymbol{R}$. Let $\mathbf{w}^{r}$ be a $|\boldsymbol{C}|$-dimensional vector whose $c$ th element $w_{c}^{r}$ is the evaluation value of the criterion $c$ from the regulating alternative $r \in \boldsymbol{R} . \mathbf{w}^{r}$ is called the weight vector for criteria from the alternative $r$. All $w_{c}^{r}$ are normalized, that is, $\sum_{c} w_{c}^{r}=1$ for all $r \in \boldsymbol{R}$. If evaluation values are consistent, all $\widehat{w}_{c}^{r\left(r^{\prime}\right)}$ are the same for all $r^{\prime} \in R$. But, almost all evaluations involve inconsistency. The set $\left\{\mathbf{w}^{r} \mid r \in R\right\}$ is transformed into $\left\{\hat{\mathbf{w}}^{r} \mid r \in R\right\}$ and the overall evaluation values of each alternative $a$ are calculated by the following iterative procedure: 


\section{Algorithm: CCM}

Step 0: For the given set $\left\{\mathbf{w}^{r} \mid r \in R\right\}$ of the weight vectors for criteria, let

$$
\mathbf{w}_{0}^{r}:=\mathbf{w}^{r}
$$

for all $r \in \boldsymbol{R}$. Let $t:=0$ and go to Step 1 .

Step 1: Let

$$
w_{t+1 c}^{i}:=\frac{1}{|R|} \sum_{r \in R} \frac{w_{t c}^{r} v_{i c} / v_{r c}}{\sum_{b} w_{t b}^{r} v_{i b} / v_{r b}}
$$

for all $i \in \boldsymbol{R}$.

Step 2: If $\max _{i \in K}\left\|\mathbf{w}_{t+1}^{i}-\mathbf{w}_{t}^{i}\right\| \leq \varepsilon$ then $\widehat{\mathbf{w}}^{r}:=\mathbf{w}_{t+1}^{r}$ for all $r \in \boldsymbol{R}$ and go to Step 3. Otherwise, update $t:=0$ and go to Step 1.

Step 3: Let $t_{a}$ denote the overall evaluation values of alternative $a$ which is the summation of products of $v_{a c} / v_{r c}$ by $\hat{w}_{c}^{r}$, where $\hat{w}_{c}^{r}$ is $c$ th element of. $\widehat{\mathbf{w}}^{r}$

$$
t_{a}=\sum_{c} \hat{w}_{c}^{r} v_{a c} / v_{r c}
$$

Kinoshita, Sekitani and Shi [3] show the convergence of CCM.

\section{Error model and GMCCM}

From formula (3), we have the following error model.

$$
\begin{gathered}
\bar{w}_{c}^{r}=c_{r^{\prime}}^{r} w_{c}^{r^{\prime}} \frac{v_{r c}}{v_{r^{\prime} c}} \varepsilon_{r r^{\prime} c} \\
\sum_{c} \bar{w}_{c}^{r}=1
\end{gathered}
$$

where $\varepsilon_{i r c}>0$ is error. Taking logarithm of (6), we have

$$
\log \bar{w}_{c}^{r}=\log c_{r^{\prime}}^{r}+\log w_{c}^{r^{\prime}}+\log \frac{v_{r c}}{v_{r^{\prime} c}}+\log \varepsilon_{r r^{\prime} c}
$$

By minimizing the sum of square of $\log \varepsilon_{r r^{\prime} c}$ 's, we have the following LLS (Logarithmic Least Square) estimate $\hat{w}_{c}^{r}$ of $\bar{w}_{c}^{r}$.

$$
\hat{w}_{c}^{r}=\frac{\left(\prod_{r^{\prime} \in R} w_{c}^{r^{\prime}} v_{r c} / v_{r^{\prime} c}\right)^{\frac{1}{|R|}}}{\sum_{b}\left(\prod_{r^{\prime} \in R} w_{b}^{r^{\prime}} v_{r b} / v_{r^{\prime} b}\right)^{\frac{1}{|R|}}}
$$

$\hat{w}_{c}^{r}$ is the geometric mean of $\widehat{w}_{c}^{r\left(r^{\prime}\right)}$ and multiplied constant of normalization as $\sum_{c} \hat{w}_{c}^{r}=1$.

All $\hat{w}_{c}^{r}$ always have the following relations.

$$
\widehat{w}_{c}^{r}=\frac{\hat{w}_{c}^{r^{\prime}} v_{r c} / v_{r^{\prime} c}}{\sum_{b} \hat{w}_{b}^{r^{\prime}} v_{r b} / v_{r^{\prime} b}}
$$

So, all $\hat{w}_{c}^{r}$ are consistent. 


\section{Conclusion}

In this study, GMCCM is found based on the multiplicative error model. GMCCM calculates the weights for the criteria with the geometric means from multi regulating alternatives without iteration. All GMCCM estimates: $\hat{w}_{c}^{r}$ are consistent

\section{REFERENCES}

[1] Saaty, T.L. (1980), Analytic Hierarchy Process, McGraw-Hill.

[2] Kinoshita, E. and Nakanishi, M. (1999),“ Proposal of new AHP model in light of dominative relationship among alternatives," Journal of the Operations Research Society of Japan, 42, 180-198.

[3] Kinoshita, E., Sekitani, K. and Shi, J. (2002),"Mathematical properties of dominant ahp and concurrent convergence method," Journal of the Operations Research Society of Japan, 45, 198-213. 